\title{
How Thalamus Connects to Spiny Stellate Cells in the Cat's Visual Cortex
}

\author{
Nuno Maçarico da Costa and Kevan A. C. Martin \\ Institute for Neuroinformatics, University of Zürich, and Swiss Federal Institute of Technology (ETH) Zürich, 8057 Zürich, Switzerland
}

In the cat's visual cortex, the responses of simple cells seem to be totally determined by their thalamic input, yet only a few percent of the excitatory synapses in layer 4 arise from the thalamus. To resolve this discrepancy between structure and function, we used correlated light and electron microscopy to search individual spiny stellate cells (simple cells) for possible structural features that would explain the biophysical efficacy of the thalamic input, such as synaptic location on dendrites, size of postsynaptic densities, and postsynaptic targets. We find that thalamic axons form a small number of synapses with the spiny stellates (188 on average), that the median size of the synapses is slightly larger than that of other synapses on the dendrites of spiny stellates, that they are not located particularly proximal to the soma, and that they do not cluster on the dendrites. These findings point to alternative mechanisms, such as synchronous activation of the sparse thalamic synapses to boost the efficacy of the thalamic input. The results also support the idea that the thalamic input does not by itself determine the cortical response of spiny stellate cells, allowing the cortical microcircuit to amplify and modulate its response according to the particular context and computation being performed.

\section{Introduction}

The receptive field (RF) transformation that occurs between the concentric center/surround RFs of thalamic relay cells of the dorsal lateral geniculate nucleus (dLGN) and the orientationselective "simple" RFs of their cortical targets in layer 4 of the primary visual cortex is a classical paradigm for cortical processing. The original and most enduring explanation is that of Hubel and Wiesel (1962), who first proposed that the simple RFs are generated solely by the convergence on a layer 4 neuron of aligned RFs of thalamic relay cells. However, this functional dominance of the thalamic input (Ferster et al., 1996; Chung and Ferster, 1998) is difficult to reconcile with structural evidence that the dLGN afferents form less then $10 \%$ of the excitatory synapses in layer 4 of area 17 of the cat (Garey and Powell, 1971; Hornung and Garey, 1981; Winfield and Powell, 1983; LeVay, 1986; Ahmed et al., 1994; da Costa and Martin, 2009a). The excitatory input to spiny stellate (SS) cells is dominated by the input from layer 6 pyramids and other layer 4 pyramids (Ahmed et al., 1994; Binzegger et al., 2004). Even though activation of single dLGN afferents in vitro evokes twofold larger EPSPs than the other known inputs to the layer 4 SS cells (Stratford et al., 1996; TarczyHornoch et al., 1999), the thalamocortical synapses undergo

Received Nov. 12, 2010; accepted Dec. 22, 2010.

This work was supported by European Union SECO Grant 216593. N.M.C. was a fellow from Fundação para a Ciencia e Tecnologia in the Gulbenkian Ph.D. Program in Biology and Medicine, grant number SFRH/BD2724/2000. John Anderson made helpful critiques of the paper. We thank Rita Bopp and German Koestinger for their expert technical assistance with the EM. We thank Anita Schmidt, Pamela Baker, and Cyrille Girardin for their help during the in vivo experiments. We also thank Dylan Muir for his help in implementing the Monte Carlo simulations.

Correspondence should be addressed to either Nuno Maçarico Costa or Kevan A. C. Martin, Institute for Neuroinformatics, University of Zürich and ETH Zürich, Wintherthurerstrasse 190, 8057 Zürich, Switzerland, E-mail: ndacosta@ini.phys.ethz.ch or kevan@ini.phys.ethz.ch.

DOI:10.1523/JNEUROSCI.5961-10.2011

Copyright $\odot 2011$ the authors $\quad 0270-6474 / 11 / 312925-13 \$ 15.00 / 0$ short-term depression that offsets this advantage in vivo (Boudreau and Ferster, 2005; Banitt et al., 2007).

How many thalamic synapses are sufficient to account for the responses of layer 4 simple cells? Our biophysically detailed simulations (Banitt et al., 2007) indicated that several hundred dLGN synapses were needed to generate multiple spiking in a simple cell with three subfields. Our most recent estimates indicate that there are only an average of between 100 and 300 thalamic synapses per SS cell (Peters and Payne, 1993; Ahmed et al., 1994; Anderson et al., 1994; Binzegger et al., 2004). This contrasts with the rat barrel cortex, where the thalamic axons (Boudreau and Ferster, 2005; Banitt et al., 2007) are estimated to form $\sim 600$ synapses with their targets (Bruno and Sakmann, 2006; but see Meyer et al., 2010).

The question we set out to answer here is whether there exist special structural features that might explain the functional dominance of the thalamic projection to layer 4 . Such structural features include a proximal position on the dendritic tree, cooperative clustering of thalamic synapses, the large size of the synapses, multiple synapses made by a single axon with its target cells [as in the retinogeniculate projection (Hamos et al., 1987) and some targets of the thalamocortical pathway (Freund et al., 1985b; da Costa and Martin, 2009b)], and whether the targets, which are mainly spines, have any characteristics that might facilitate or augment synaptic current flow. Physiological studies indicate that virtually all neurons in layer 4 can be driven monosynaptically from the dLGN, which implies that all simple cells receive a similar weight of input from dLGN. However, the physiology does not give information about the number of synapses or the structural specializations outlined here, which can only be assayed by direct analyses of the structure.

In previous quantitative work on mapping the entire thalamocortical input to layer 4 neurons, we used indirect morphological 
criteria to identify putative thalamic synapses (Ahmed et al., 1994, 1997). Given the importance of understanding the nature of the thalamocortical transmission, we have now used tracers to identify directly the thalamocortical synapses and thus determine whether there are special structural features that could explain their physiological dominance.

\section{Materials and Methods}

Surgical procedures. All experiments, animal treatment, and surgical protocols were carried out with authorization and under license granted to K.A.C.M. by the Cantonal Veterinary Office of Zürich, Switzerland. The material presented here originates from three cats. One of the cats was 9 weeks old and the other two were adults with ages of 9 and 19 months. Each experiment was composed of two surgeries. During the first surgery the neural tracer biotinylated dextran amine (BDA), 10,000 molecular weight (Invitrogen), was injected into the dLGN of the cat. After the survival period that lasted 5,7 , and $22 \mathrm{~d}$, a second surgery was performed where we recorded and intracellularly labeled neurons in area 17.

All cats were prepared for surgery after the administration of subcutaneous or intramuscular injection of xylazine $\left(0.5 \mathrm{mg} \cdot \mathrm{kg}^{-1}\right.$; Rompun, Beyelar) and ketamine $\left(10 \mathrm{mg} \cdot \mathrm{kg}^{-1}\right.$; Narketan 10, Vétoquinol), and additional gas anesthesia using $1-2 \%$ halothane (Arovet) in oxygen/nitrous oxide $(50 / 50 \%)$ was also delivered. After induction of anesthesia, some of the cats received a subcutaneous injection of atropine (Graeub) of $0.05 \mathrm{mg} \cdot \mathrm{kg}^{-1}$ to decrease secretions. All experiments were performed under sterile conditions. The femoral vein was cannulated and alphaxalone/alphadalone (Saffan, Glaxo) was delivered to maintain general anesthesia during the remainder of the experiment. The cat was intubated and moved to a stereotaxic apparatus where it was respirated artificially with a mixture of oxygen/nitrous oxide $(30 \% / 70 \%)$. The end-tidal $\mathrm{CO}_{2}$ was maintained at $\sim 4.5 \%$. Four percent lidocaine gel (G. Streudi) was applied to all pressure points. Intravenous anesthesia was supplemented with halothane gas if required during the remainder of the experiment.

Electroencephalogram (EEG), electrocardiogram, end-tidal $\mathrm{CO}_{2}$, and rectal temperature were monitored continuously during the entire experiment. A thermistor-controlled heating blanket maintained the cat's rectal temperature at $37^{\circ} \mathrm{C}$. Topical antibiotics (Voltamicin, Novartis) and $1 \%$ atropine Novartis) (to paralyze accommodation) were applied to the eyes before they were covered with gas-permeable contact lenses. Five percent phenylephrine (Blache) was applied to each nictitating membrane to retract them.

After surgery, the cats were given an intravenous injection of the muscle relaxant gallamine triethiodide (12 mg induction dose, $5 \mathrm{mg}$. $\mathrm{kg}^{-1} \cdot \mathrm{h}^{-1}$ thereafter) (Flaxedil, May Balces). This low dose of muscle relaxant was enough to reduce eye movement during the short period of time that electrophysiological recording was made to locate individual layers of the dLGN. Delivery of the muscle relaxant was stopped 2-3 $\mathrm{h}$ before the end of the electrophysiological recording and injections of the tracer.

After iontophoresing the neural tracer BDA $(10,000 \mathrm{MW})$ into the dLGN, the craniotomies were closed by gluing back the original bone flap to the skull using dental cement. A prophylactic dose of antibiotics was administered to the cat both intramuscularly $\left(10 \mathrm{mg} \cdot \mathrm{kg}^{-1}\right.$, Clamoxyl, GlaxoSmithKline) and topically on the skull (Soframycin, Aventis). The skin and head muscles were sutured, and respiration and muscular tonus were monitored to assess the animals' recovery. In the two days that followed surgery, the cat received a subcutaneous injection $10 \mathrm{mg} \cdot \mathrm{kg}^{-1}$ Clamoxyl per day. During the survival period, the cat was monitored daily to assess whether any signs of pain or infection were visible. If needed, a dose of analgesic $\left(0.01 \mathrm{mg} \cdot \mathrm{kg}^{-1}\right.$ buprenorphinum; Tengesic, Essex Chemie) was administered.

After the survival period, the cats were anesthetized and the surgery was performed as previously described with a few extra procedures. The femoral artery was cannulated to measure blood pressure, and a tracheotomy was also performed. The cat was paralyzed with a continuous infusion of gallamine triethiodide $\left(13 \mathrm{mg} \cdot \mathrm{kg}^{-1} \cdot \mathrm{h}^{-1}\right)$ and $(+)$ tubocurarine chloride hydrate $\left(1 \mathrm{mg} \cdot \mathrm{kg}^{-1} \cdot \mathrm{h}^{-1}\right)$ (Sigma). The eyes were then refracted and lenses were added to focus the eyes on a tangent screen positioned at $114 \mathrm{~cm}$.

Injections of $B D A$ in the A lamina of the $d L G N$. A small craniotomy was performed over area 17 (Horsley-Clark coordinates anteroposterior (AP) -3 to -5 , and mediolateral (ML) $0-2$ ) and over the dLGN (centered at Horsley-Clark coordinates AP 8 to 9 and ML 5 to 6) with a trephine. A glass micropipette filled with $1 \mathrm{~m} \mathrm{KCl}$ was advanced into area 17 , and the position, orientation, and eye dominance of receptive fields were noted. Afterward a tungsten electrode insulated with epoxy was positioned in the center of the craniotomy over the dLGN, and after opening a slit in the dura mater it was lowered into the brain. Once the dLGN was found, the electrode position was moved according to the retinotopic map of Sanderson (1971) until the receptive field position of the dLGN neurons of the A lamina was superimposed with that recorded from area 17 . Then, the position and depth of the electrode were noted and the tungsten electrode was replaced by a glass micropipette filled with a solution of BDA (10,000 MW; Invitrogen). The pipette tip diameter varied from 10 to $18 \mu \mathrm{m}$. The A lamina was then found again by recording through the injection pipette. Injections were made iontophoretically lasting $15-20 \mathrm{~min}$, with current pulses of $3 \mathrm{~s} \mathrm{on} / 3 \mathrm{~s}$ off and amplitude of $2-4 \mu \mathrm{A}$. The BDA was delivered as a $10 \%$ solution in $0.01 \mathrm{M}$ phosphate buffer (PB), pH 7.4, or $0.01 \mathrm{M} \mathrm{PB}$ and $0.2 \mathrm{M} \mathrm{KCl}$ or $0.05 \mathrm{M}$ Tris and $0.2 \mathrm{M} \mathrm{KCl}$. Injections were separated by at least $500 \mu \mathrm{m}$, usually more, so that the retinotopic progression could be distinguished in the cortex.

Cortical recordings and intracellular labeling. Glass micropipettes were beveled to an impedance of between 40 and $100 \mathrm{M} \Omega$ and filled with a $4 \%$ solution of horseradish peroxidase (HRP, Roche) in $0.05 \mathrm{M}$ Tris and $0.2 \mathrm{M}$ $\mathrm{KCl}$ at $\mathrm{pH}$ 7.9.

A craniotomy was performed over area 17 from Horsley-Clark coordinates $\mathrm{AP}-2$ to -7 and $\mathrm{ML}$ from the midline to $3 \mathrm{~mm}$ lateral, and a plastic chamber was mounted over the craniotomy and fixed to the bone with dental cement. A small durotomy was made for each penetration. The location of the penetration was noted on a drawing of the pattern of blood vessels on the surface of the cortex. Before the micropipettes were lowered into the chamber, it was filled with agar (Sigma) in Ringer solution and sealed with paraffin.

The neurons were recorded extracellularly and their receptive fields were plotted by hand on a tangent screen. When possible, the following receptive field properties were recorded: ocular dominance, orientation, direction preference/selectivity, receptive field size, subfields, and type (simple or complex). An attempt was then made to impale the neuron and inject HRP intracellularly. This was done by advancing the micropipette in $2 \mu \mathrm{m}$ intervals while passing current pulses of $1-2 \mathrm{nA}$. As soon as a neuron was impaled, HRP was ionophoresed into it. This was done by passing positive pulses of $2-5 \mathrm{nA}$ in a $200 \mathrm{~ms}$ on/50 ms off duty cycle for a duration that ranged from $5 \mathrm{~s}$ to $\sim 4 \mathrm{~min}$. The cell was considered impaled if there was a drop of DC potential (in our case from -20 to -70 $\mathrm{mV}$ ), the amplitude of the action potential became very large, and subthreshold activity appeared. A detailed description of the method is presented in Martin and Whitteridge (1984). Because of the uncertainty about the exact location of the labeled thalamic afferents in the cortex and the low yield in term of the numbers of recovered SS neurons per experiment, we performed several penetrations over a large craniotomy. This procedure compromises the stability of the intracellular recordings and only rarely we could verify the receptive field properties while recording intracellularly. In a previous study (Martin and Whitteridge, 1984), neurons were recorded both intracellularly and extracellularly and the consistency of the receptive field properties between the two recording conditions was confirmed.

Perfusion. At the end of the acute experiment the cat was given an extra dose of anesthesia until the EEG became flat and the end-tidal $\mathrm{CO}_{2}$ was lowered to $3.5 \%$. The cat was then perfused transcardially with normal $0.9 \% \mathrm{NaCl}$ solution followed by a warm solution of $4 \%$ paraformaldehyde $(\mathrm{w} / \mathrm{v}), 0.3 \%$ glutaraldehyde $(\mathrm{v} / \mathrm{v})$, and $15 \%$ saturated solution of picric acid (v/v) in $0.1 \mathrm{M} \mathrm{PB}, \mathrm{pH}$ 7.4. After fixation, the cat was perfused with a 10 and $20 \%$ solution of sucrose in $0.1 \mathrm{M} \mathrm{PB}$. The brain was stereotaxically cut and blocks containing the injected dLGN and area 17 were removed. 

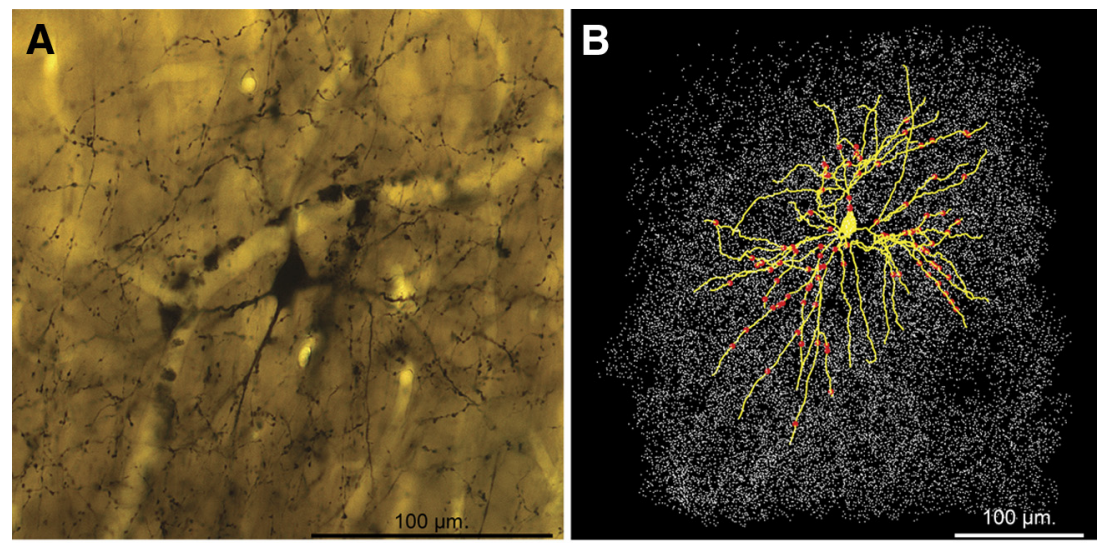

C

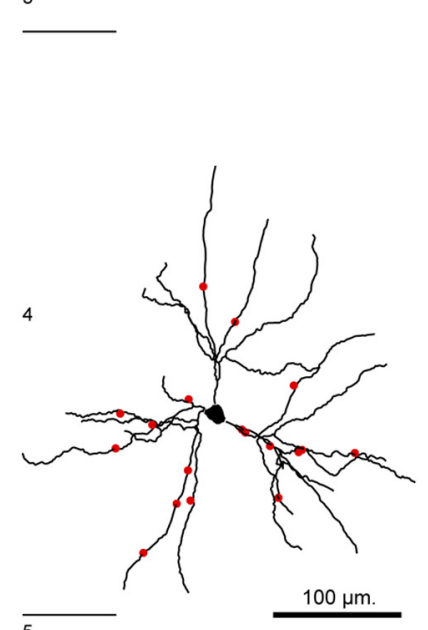

D 3

E

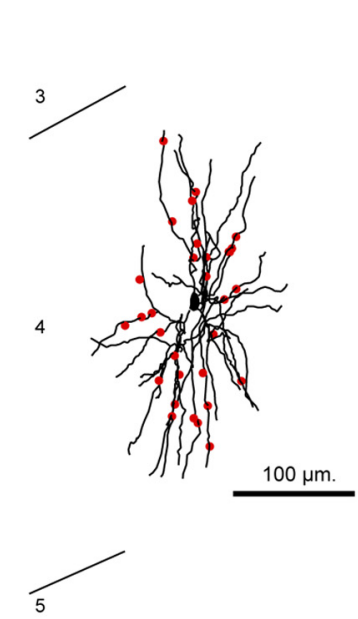

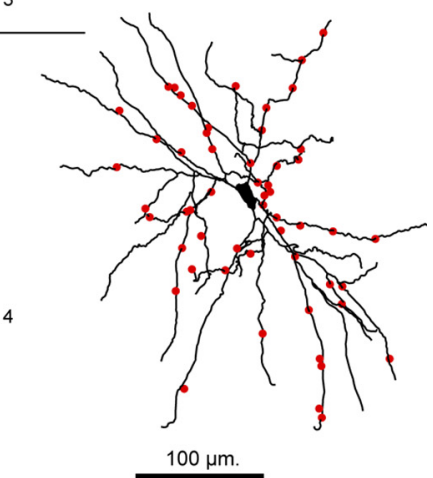

$\mathbf{F}$

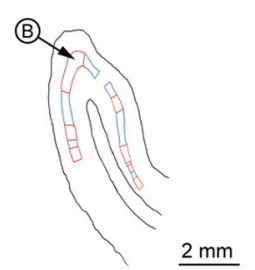

G

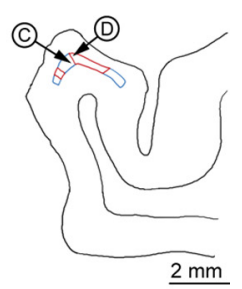

$\mathbf{H}$

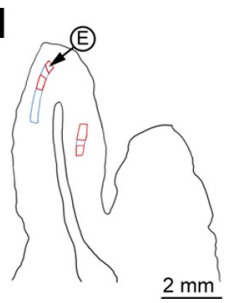

Figure 1. Spiny stellate cells. $\boldsymbol{A}$, Light micrograph of spiny stellate cell surrounded by dLGN axons. $\boldsymbol{B}$, LM reconstruction of the spiny stellate cell in $\boldsymbol{A}$, with dendrites in yellow and contacts with the dLGN boutons and axons represented by red dots. The white dots are all of the dLGN boutons judged by LM that surrounded the neuron's dendrites. This neuron had a simple receptive field, $0.25^{\circ} \times 0.1^{\circ}$ (length $\times$ width), direction preference, and ocular dominance group 2. $\boldsymbol{C}-\boldsymbol{E}$, LM reconstruction of the dendrite and soma (black) of the remaining three spiny stellate neurons, with LM contacts between dendrites and dLGN axons and boutons shown in red. Layers $(3,4,5)$ are shown to the left. $\boldsymbol{F}-\boldsymbol{H}$, Outline of coronal sections with arrows indicating the location of each neuron; outlined in red are the contralateral axonal patches in layer 4 and the regions of weak thalamic labeling are outlined in blue, representing the ocular dominance patch of the ipsilateral eye. C, G, Neuron Cat1804-P4C4 responded preferentially to the ipsilateral eye. D, G, Neuron Cat1804-P3C4. E, H, Neuron Cat2003-S28C1. F, Neuron Cat0904-P4C2 responded preferentially to the contralateral eye.

Histology. The blocks of brain were allowed to sink in sucrose solutions of 20 and $30 \%$ in $0.1 \mathrm{M}$ PB to provide cryoprotection. The blocks were then freeze thawed twice in liquid nitrogen to enhance the penetration of immunochemicals by mechanically disrupting the tissue. The brain blocks were then washed in $0.1 \mathrm{M}$ PB for at least $2 \mathrm{~h}$ to allow them to recover from the shrinkage provoked by the incubation in sucrose solution. Sections were cut from the blocks at $80 \mu \mathrm{m}$ in the coronal plane and collected in $0.1 \mathrm{M} \mathrm{PB}$. After cutting, the sections were washed several times in buffer to remove any remaining fixative. To reveal BDA, the sections were washed in TBS and then incubated overnight $\left(5^{\circ} \mathrm{C}\right)$ with an avidin-biotin complex (Vectastain Elite ABC kit, Vector Laboratories). The peroxidase activity was identified using 3,3' -diaminobenzidine tetrahydrochloride (DAB) with nickel intensification. After assessment by light microscopy (LM) regions of tissue were selected for subsequent electron microscopy (EM) analysis. These sections were treated with $1 \%$ osmium tetroxide in $0.1 \mathrm{M} \mathrm{PB}$, dehydrated through alcohols (1\% uranyl acetate in the $70 \%$ alcohol) and propylene oxide, and flat mounted in Durcupan (Fluka) on glass slides. Regions with dense dLGN labels were blocked and cut into ultrathin sections of $60 \mathrm{~nm}$ and the sections were then collected onto Pioloform-coated copper grids. Sections not selected for EM were air dried, dehydrated through alcohols, and mounted with DPX (Fluka). Sections including the dLGN were stained using neutral red to visualize the different laminae.

Light microscopy. Neurons and axons of interest were reconstructed first in two dimensions using a drawing tube attached to a light microscope and then in three dimensions using an in-house three-dimensional (3-D) reconstruction system (TRAKA) (Botha et al., 1987). A toolbox was designed in Matlab to import, analyze, organize, plot, and manage in a database the 3-D reconstructions from TRAKA. The locations of LM contacts between dLGN terminals and spiny stellate neurons were noted on LM 3-D reconstructions. An LM contact was marked whenever a gap between a labeled dLGN axon and a labeled dendrite could not be discerned at the LM level, making the two labeled structures appear contiguous. All contacts were identified with a magnification of $\times 1000$ under oil, using a Leica DM 2500 microscope and with a $100 \times$ objective (aperture 1.30).

Electron microscopy. After making the LM observations of labeled axons, labeled neurons and LM contacts between dLGN axons and SS dendrites, we prepared regions of interest to be observed with transmission electron microscopy. Serial ultrathin sections were collected at $60 \mathrm{~nm}$ thickness on Pioloform-coated single slot copper grids.

Synapse classification and measurements. Synapses and associated structures were classified using conventional criteria (Gray, 1959; Colonnier, 1968). For a LM contact to be classified as an EM-confirmed synapse, it had to form a postsynaptic density (PSD) separated by a synaptic cleft from the presynaptic termi- 
A

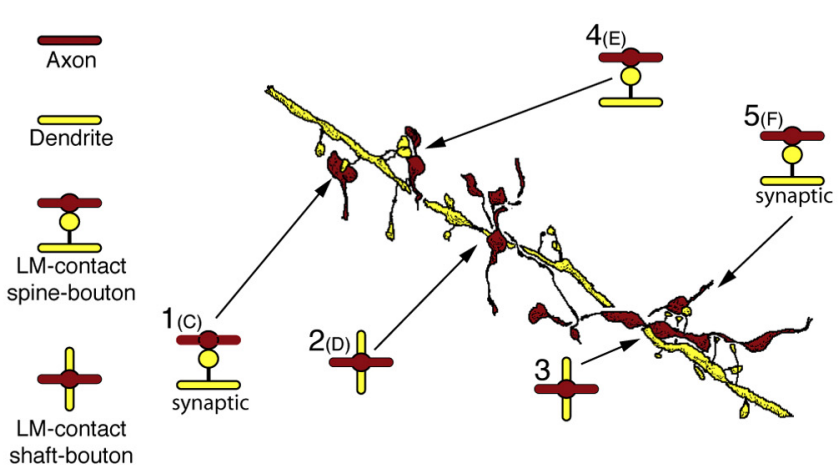

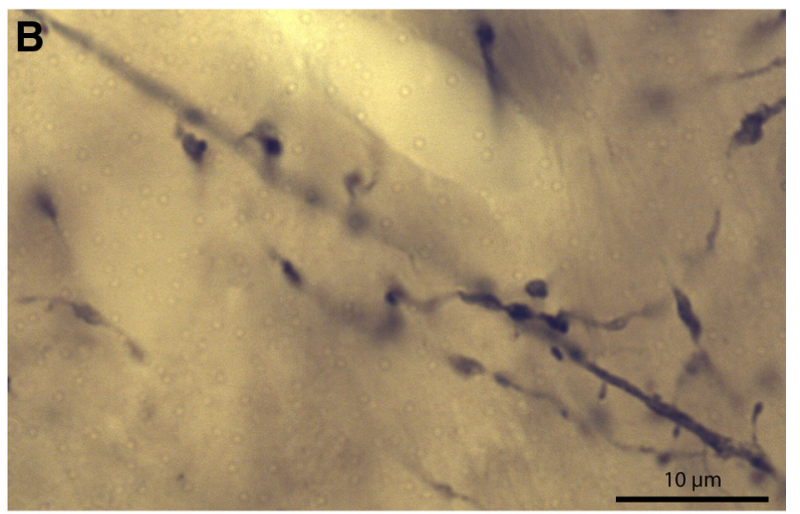
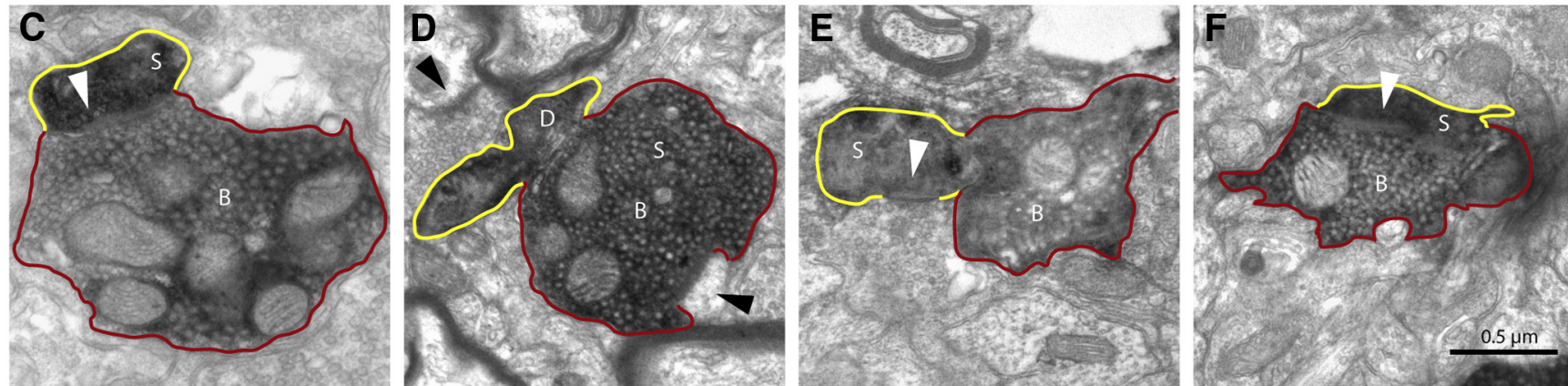

Figure 2. Contacts between spiny stellate cell and dLGN boutons seen by LM and EM. $A$, Drawing of segments of spiny stellate dendrite and dLGN boutons making contacts with them. EM examination showed that contacts 1 and 5 formed synapses while the others did not. Using EM we discovered that contact 3 had a gap between the dendrite and the bouton. $\boldsymbol{B}$, Light micrograph of the dendritic segment shown in $\boldsymbol{A}$. Scale bar, $10 \mu \mathrm{m}$. $\boldsymbol{C}-\boldsymbol{F}$, Electron micrographs of $L M$ contacts $1,2,4$, and 5, respectively. The labeled dLGN bouton (B) contacted a labeled spine (S) or dendritic shaft (D). Black arrowheads indicate synapses formed with unlabeled postsynaptic structures. White arrowheads indicate synapses formed with labeled spines. Scale bar, $0.5 \mu \mathrm{m}$.

nal, where synaptic vesicles had to be observed in close proximity or in contact with the presynaptic membrane. If the synaptic cleft was difficult to identify because of the shape of the synapse or the angle of sectioning, the sections were tilted in the EM to provide a better view.

The area of the postsynaptic density,

$$
\text { PSD area }=\sum_{\text {All sections }}\left(\text { PSD length } \text { section } \mathrm{i} \times \text { section thickness }_{\text {section } \mathrm{i}}\right),
$$

was calculated as the sum of the products of the length of the PSD in a single section and the section thickness. Measuring synaptic areas when the postsynaptic terminal is labeled might lead to an underestimation of its value, because the dark DAB deposit might obscure part of the PSD. However, it is unlikely that this affects our comparison, since both populations of reconstructed spines (targeted by dLGN boutons and unlabeled boutons) were labeled.

The distance between an LM contact and the soma was measured from the path length of the dendrite taken from the 3-D LM reconstruction.

3-D reconstructions. Collections of serial sections were aligned, digitized, and reconstructed using either Reconstruct (Fiala, 2005) or TrakEM2 (Cardona et al., 2010). We mostly used translation and rotation movements for alignment of sections of the same magnification. Very rarely, when a strong deformation was present in the section we used scale and shear movements to align the reference points. Alignment was done in a serial way with every section being aligned with the preceding one. When a section had to undergo scaling or shear we aligned the two adjacent sections with each other instead of with the deformed photograph. The reconstructed profiles were rendered using the open source software Blender (Stitching Blender Foundation).

LM contact analysis. LM contacts were examined by EM to investigate synaptic connectivity between the dLGN axon and the labeled cortical neurons. Blood vessels, labeled dendrites, and axons that were visible both at the LM and EM levels were used to find the contacts in the ultrathin sections. Once the LM contact was found, we verified whether there was indeed a synapse between the two-labeled structures or not.
Usually a high-power EM photograph $(33,000 \times, 25,000 \times$, or $19,000 \times)$, a low-power EM photograph $(3000 \times$ to $7900 \times)$, and a light micrograph were superimposed with each other to confirm the location of the contact in the EM. In some cases the presynaptic and postsynaptic structures were reconstructed from serial electron micrographs and the reconstruction was superimposed on the light micrograph of the contact. The LM contact was initially sampled randomly from each neuron. At a later stage a set of 12 extra LM spine contacts was sampled from neuron Cat0904P4C2.

Physical disector. The percentage of labeled (dLGN) synapses in the neuropil surrounding the dendrites of the reconstructed SSs was estimated using the physical disector method (Sterio, 1984). Synapses were classified using the same criteria as in da Costa and Martin (2009a). The sampling area was defined by a polygon formed by the connection of the distal tips of the dendrite. The disector is composed of two serial sections of known thickness $(\sim 60 \mathrm{~nm})$. Reference and lookup section were separated by one intervening section. The labeled dLGN synapses were a very rare event in the neuropil and so we developed a systematic random sampling scheme (Gundersen and Jensen, 1987; Slomianka and West, 2005) optimized for rare events (da Costa et al., 2009). The disectors had a size of $5 \times 5 \mu \mathrm{m}$ and were collected by taking photographs at a magnification of $13,500 \times$ with a digital camera (11 mega pixels, Morada, Soft Imaging Systems). Briefly, we used a systematic random sampling scheme but only took actual photographs of sample sites that had a synapse formed by a labeled bouton in the reference section. The sites that did not have a labeled profile with a synapse were noted, but no photograph was taken and no synapse was counted. The mean density of labeled synapses was calculated using the volume of all the sampling sites, including those that were not photographed (where no dLGN synapse was found). The mean density of all synapses in the neuropil could only be calculated using the sampling sites that were photographed.

Estimation of the number of dLGN synapses per spiny stellate cell. We estimated the number of dLGN synapses per neuron by the following equation: 


\section{A}
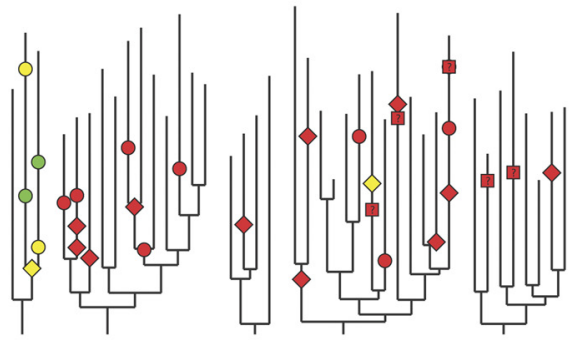

C2003S28C1

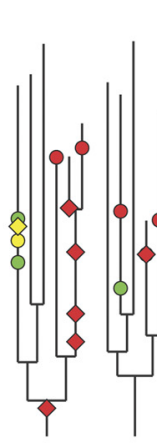

C1804P3C4
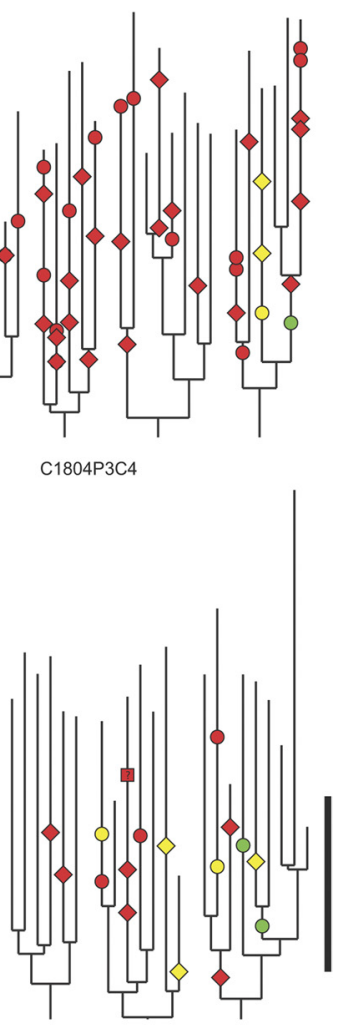
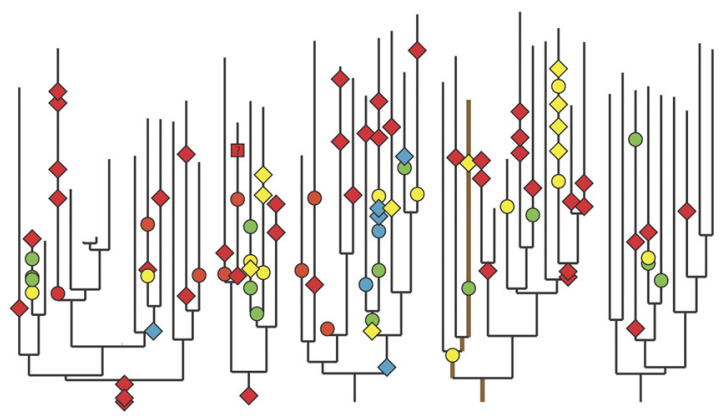

C0904P4C2

$\diamond$ shaft

0 not verified at EM $O$ synapse

$>$ no synapse
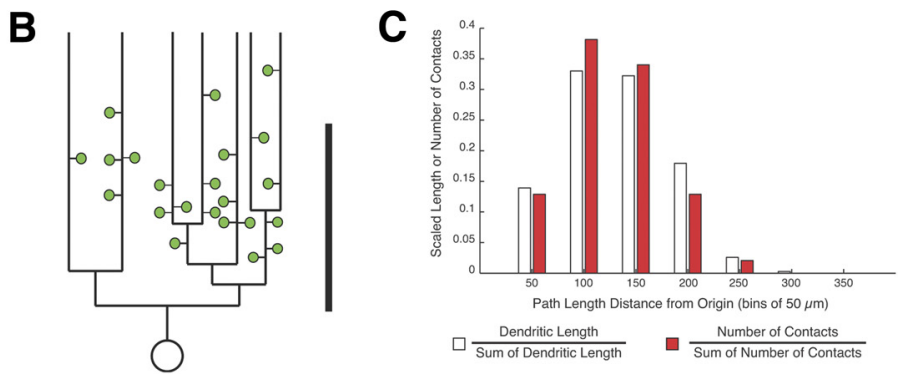

Figure 3. Location of the dLGN contacts on spiny stellate cells. $A$, Dendrograms showing the location of the dLGN contacts on the four spiny stellate cells used in this study. The dendrite of neuron Cat0904-P4C2 (brown) was also reconstructed from EM serial sections as is shown in Figure 6. Contacts rated "undetermined" (blue dots) were unclear or damaged when observed by EM, and the presence of synapses could not be determined. Scale bar, $100 \mu \mathrm{m}$. B, Summary of the location of the actual dLGN synapses. Each synapse is plotted as a "spine" at the path-length distance to the soma and branch order. The distance between the root and the tip of a dendrite in the dendrogram is the mean tip to root path length distance of a SS dendrite. Most of the synapses were formed with terminal segments of dendrite. Each thalamic synapse was indicated as a green spine, and the soma as a white circle. C, Histogram showing the distribution of dendritic length of all SS and number of all dLGN LM contacts with distance to the soma. The scaled dendritic length is shown in white (length in bin/total length) and the scaled number of contacts in red (contacts in bin/total contacts). The LM contacts occur in proportion to the available length of dendrite.

$$
\left(n_{\mathrm{c}} \times c_{\text {syn }}\right) \times\left(\mathrm{dLGN}_{\mathrm{t}} / \mathrm{dLGN}_{\mathrm{l}}\right),
$$

where $n_{c}$ is the number of LM contacts per neuron, $c_{\text {syn }}$ is the proportion of LM contacts that form synapses (varies between 0 and 1 ), dLGN $\mathrm{d}_{\mathrm{t}}$ is the percentage of asymmetric synapses that are provided by all the dLGN axons. The dLGN $\mathrm{t}_{\mathrm{t}}$ was calculated individually for each one of the cats in a separate study (da Costa and Martin, 2009a) and is in agreement with other previous estimations (LeVay, 1986; Peters and Payne, 1993; Ahmed et al., 1994). dLGN 1 is the percentage of asymmetric synapses that we labeled around the dendrite with our dLGN injections.

Calculation of the error of the estimation. For each sampled value the error of the estimation was expressed as the SEM. For the calculation of error of the probability of an LM contact forming a synapse, we used a

binomial distribution. This implies that we are able to detect all contacts (which we controlled with the dendrite EM reconstruction and by having several observers examining the dendrites at LM) and can accurately confirm whether an LM contact forms a synapse. The errors were then propagated in quadrature to obtain an estimation of the error of the number of dLGN synapses formed with each neuron.

Simulations of random locations of thalamic synapses. A Monte Carlo analysis was made to test whether thalamic synapses are randomly distributed over the dendritic arbor of SS cells or whether synapse locations show evidence of clustering. Test synapses were pseudorandomly located on dendrograms of each of the four SSs. The distance from the soma to each test synapse was normally distributed, with a mean and SD matching that of the experimentally observed thalamic synapses. The number of test synapses constructed for each SS was determined by estimating the total number of thalamic synapses expected to be made with the SS, as measured in this study. To approximate the measurement technique used here, a subset of the randomly located synapses was sampled for analysis. The number of these test synapses in the sample, $n^{\mathrm{SSi}}$, varied for each neuron SSi and was calculated using the following formula:

$$
n^{\mathrm{SSi}}=n_{\mathrm{syn}}^{\mathrm{SSi}}+p_{\mathrm{sp}} \times \text { LMcontact }_{\mathrm{sp}}^{\mathrm{SSi}},
$$

where $n_{\text {syn }}^{\text {SSi }}$ is the number of dLGN EMconfirmed synapses formed on neuron $\mathrm{SSi}, p_{\mathrm{sp}}$ is the probability that an LM contact between a bouton and a spine forms a synapse (which in this study we found to be 0.55), and $\mathrm{LMcontact}_{\mathrm{sp}}^{\mathrm{SSi}}$ is the number of bouton-spine contacts observed by LM that were not investigated by EM. The simulation was repeated 10,000 times for each neuron. All simulations were performed in Matlab (MathWorks).

For each randomly generated test synapse, the distance to the nearest test synapse was collected. The average of these measurements formed the test statistic for a single set of randomly generated test synapses for a single SS cell. The same measurement was made for experimentally observed thalamic synapses. For each SS, the set of thalamic synapses was considered to consist of all thalamic synapses confirmed at EM, plus a random subset of unverified thalamic bouton-spine contacts. This set was constructed by considering in turn every possible combination of $55 \%\left(p_{\mathrm{sp}}\right)$ of unverified thalamic bouton-spine contacts and declaring them to be true thalamic synapses. A test statistic was therefore generated for every possible combination of unverified bouton-spine contacts for each SS.

A $p$ value was estimated for each combination of potential thalamic synapses for each SS under the null hypothesis that thalamic synapses are randomly located over SS dendritic arbors. The $p$ value estimate was given by the proportion of measurements from sets of random test synapses that were equal to or smaller than the measurement made over potential thalamic synapses.

Statistical analysis. Statistical analysis was performed in Matlab (MathWorks). The Lillie test was used to determine whether the data were normally distributed. A $t$ test was used when the data were normally 
distributed, and otherwise a Wilcoxon rank sum test was used. The Kolmogorov-Smirnov test was used to determine whether the distribution of length of dendrite was significantly different from the distribution of LM contacts.

\section{Results}

Labeling of dLGN axons and their spiny stellate cortical targets

The thalamic afferents of area 17 of three cats were labeled with BDA (10,000 MW) after injections in the dLGN. The results presented here were taken from the same animals used in our study on the total percentage of asymmetric synapses formed by the thalamocortical pathway (da Costa and Martin, 2009a). In most cases the injections were confined to the contralateral A lamina (da Costa and Martin, 2009a). Labeled axons were found in areas 17 and 18 , where they formed arbors mostly in layers 4 and 6. Axons labeled by dLGN injections always formed patches in area 17 that represent the contralateral eye. As documented previously (LeVay et al., 1978), some thalamic boutons were also found in the adjacent regions representing the ipsilateral eye (Fig. 1). Part of the ipsilateral label is attributable to thalamic boutons formed between ocular dominance clusters (Freund et al., 1985a; Humphrey et al., 1985), and in some cases also to spillover of the injection site to lamina A1.

Because of the difficulty of labeling neurons in the same region of cortex where the labeled thalamic afferents were located, we directed our efforts to injecting intracellularly as many cells as possible and recovered 12 neurons in layer 4 from the 150 recorded, four of which were SSs surrounded by dLGN boutons. The eight others were inhibitory neurons, star pyramidal cells, and SSs located outside the regions of labeled thalamic axons and will not be considered in this study. We made extracellular and intracellular recordings from the four orientation-tuned SSs, and in three cases plotted their RFs. The neuron shown in Figure 1 A, B (C0904P4C2) had a simple receptive field that is typical of SSs (Martin and Whitteridge, 1984), and its ocular dominance matched the SS's location in a cluster of dLGN axons representing the contralateral eye. A SS dominated by the ipsilateral eye is shown in Figure $1 C$; as expected for its ocular dominance, it lies outside the main cluster of labeled dLGN axons.

Light microscopic identification of putative sites of synapses between dLGN axons and SS dendrites

Contacts between SS dendrites and dLGN axon were observed by LM for all of the neurons. We scored an LM contact when no gap could be seen between a labeled dLGN axon and a labeled dendrite, as assessed using oil immersion (magnification of $\times 1000$ ). We included observations of LM contacts between a dendritic shaft or spine and a dLGN bouton or axon. In Figure 2 (see also Figs. 4, 5) we show LM photographs and drawings of some of

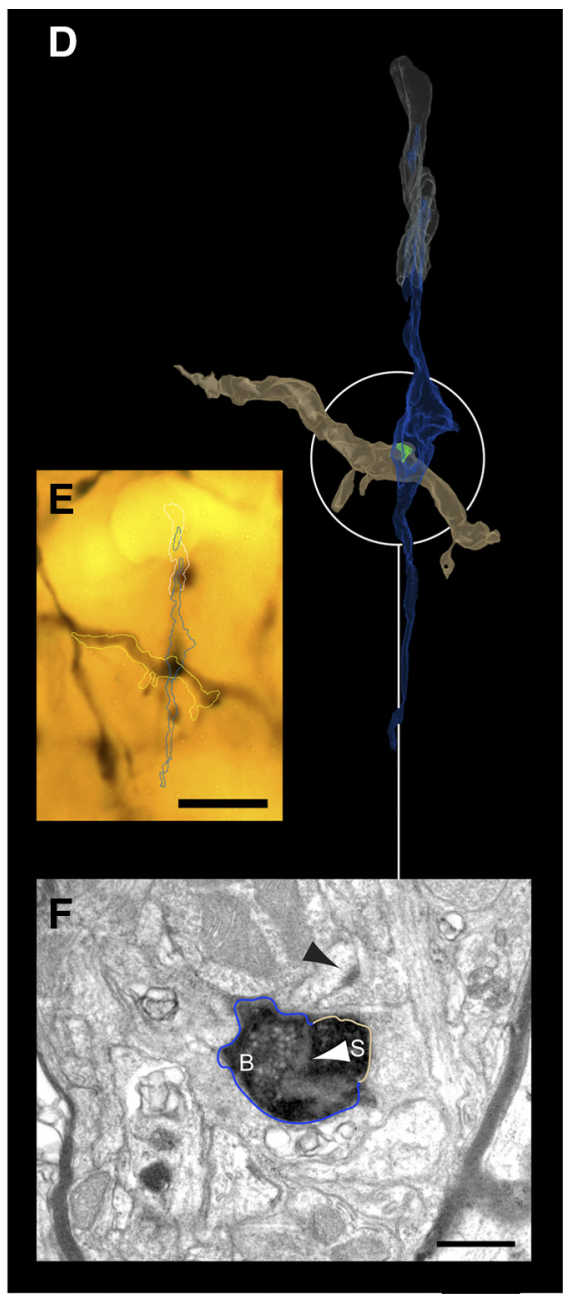

Figure 4. LM contacts that formed synapses between $d L G N$ boutons and the spines of two spiny stellate cells. $A, D, E M$ brown, and synapses are in green. $\boldsymbol{B}, \boldsymbol{E}$, Light micrographs showing the contact with superimposed EM reconstruction. Scale bar, 10 $\mu \mathrm{m} . \boldsymbol{C}, \boldsymbol{F}$, Electron micrographs showing the labeled dLGN bouton (B) forming a synapse (white arrowheads) with a labeled spine (S). Black arrowheads indicate synapses between unlabeled profiles. Scale bar, $0.5 \mu \mathrm{m}$.

these LM contacts. On a few occasions there were several labeled boutons from different axons around a particular region of the dendrite, making it impossible to segment the structures and to identify at LM which particular axons were contacting the dendrite. These "hot spots" were recorded as a single LM contact. A total of $191 \mathrm{LM}$ contacts were recorded.

The SS whose dendrites had the most LM contacts from the dLGN axons is shown in Figure $1 A$. A reconstruction of the same neuron is shown embedded in the cloud of all the labeled dLGN boutons (white dots) that surrounded it (Fig. 1B). The sites of LM contacts with the dendrite of the SS are indicated by the red dots. The locations of the dLGN LM contacts with the remaining SSs are also shown in Figure 1 and in the dendrograms of Figure $3 A$. LM contacts were made with all the dendrites of all the neurons both proximally and distally. There was no apparent preference for very proximal locations. The number of LM contacts at a particular distance to the soma was proportional to the available dendrite. This is evident quantitatively when the distribution of the path length of dendrites of all the SSs is compared with the distribution of LM contacts (Fig. 3C). The two distributions (length of dendrite and number of LM contacts) were not significantly different from each other in the 

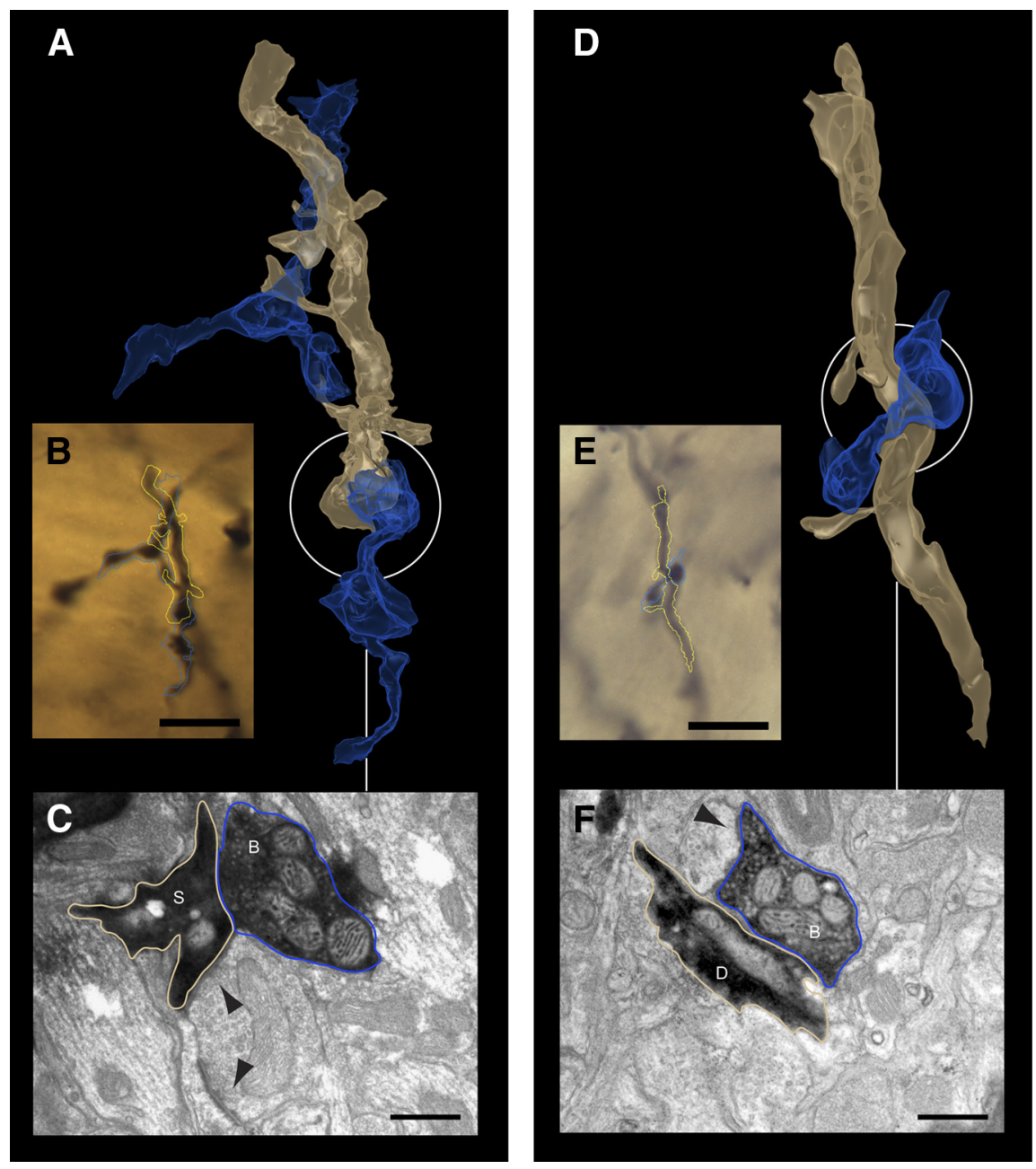

Figure 5. Contacts between $d L G N$ boutons and dendritic spines that did not form synapses. $\boldsymbol{A}-\boldsymbol{F}$, The $L M$ and serial $E M$ reconstructions of labeled axon and dendrites are shown for a spine contact $(\boldsymbol{A}, \boldsymbol{B})$ and a dendritic shaft contact $(\boldsymbol{D}, \boldsymbol{E})$. dLGN axons are shown in blue, and dendritic spines and shafts are shown in light brown $(\boldsymbol{A}, \boldsymbol{D})$. Light micrographs show the contact $(\boldsymbol{B}, \boldsymbol{E})$. Scale bar, $10 \mu \mathrm{m}$. Electron microphotographs $(\boldsymbol{C}, \boldsymbol{F})$ showing the labeled dLGN bouton (B), labeled dendritic spine (S), and shaft (D). Arrowheads indicate synapses. Scale bar, $0.5 \mu \mathrm{m}$.

pooled data of Figure $3 C$ (Kolmogorov-Smirnov test; $p=$ 0.5116) or in any of the cells (Kolmogorov-Smirnov test; Cat2003S28C1, $p=0.8660$, Cat1804P3C4, $p=0.8540$, Cat1804P4C4, $p=0.2556$; Cat0904P4C2, $p=0.7967)$.

The LM contacts were mostly made with dendritic shafts (56\%), but a significant proportion of contacts were made with dendritic spines (42\%). In $2 \%$ of the cases the postsynaptic structure was unresolved by LM.

Although occasional retrogradely labeled corticothalamic cells were seen (da Costa and Martin, 2009b), none were located in the proximity of the SS used in this study. Moreover, none of the axons contacting the SS resemble those of layer 6 pyramidal cells, which are studded with small bouton terminaux (Martin and Whitteridge, 1984; McGuire et al., 1984).

\section{Do the LM contacts mark the sites of synapses?}

All four SS cells were sectioned into ultrathin sections and at least $20 \%$ of the LM contacts in each were investigated by correlated LM and EM. The locations of the LM contacts examined with EM are shown in the dendrograms of Figure 3A.

Initially, $50 \mathrm{LM}$ contacts were examined in the EM, and of these 14 formed synapses, 30 did not, and 6 were unclear or damaged in the preparation so that the existence of synapses at the point of contact could not be determined. We also found a synapse between the dLGN axon and the SS that we had not identified as a contact in the LM. In this synapse the dLGN boutons obscured the LM visualization of the spine head, and the thin spine neck was also missed. In total, only $32 \%$ of the LM contacts proved to be synapses. For the EM analysis of LM contacts we investigated 20 LM contacts occurring with spines, 19 with shafts, and 5 whose target was unclear at LM. All of the thalamic boutons formed synapses with dendritic spines (Fig. 4). White and Rock (1980) found the same result in their reconstruction of thalamic input to a single SS cell in the mouse somatosensory cortex. All of the spines targeted by thalamic boutons were of the "mushroom" type, which is quite unlike the rat auditory cortex where the thalamic synapses form with "stubby" spines and produce on average larger EPSPs than nonthalamic synapses with mushroom spines (Richardson et al., 2009).

Of the 30 LM contacts that did not form a synapse when examined in the EM (Figs. 3A, 5), 15 of them were not even in physical contact with the labeled SS. In the other 15 cases the membranes of the twolabeled structures were in physical contact, but they did not form a synapse with each other. With the exception of one bouton, thalamic boutons that made nonsynaptic LM contacts with labeled spines or dendrites always formed synapses with other unlabeled targets. Conversely, whenever an LM contact with a spine did not form a synapse, the same spine formed a synapse with an unlabeled bouton with the exception of one spine, which formed no synapse at all.

After this initial analysis, an additional 12 LM contacts from neuron Cat0904P4C2 were examined at the EM level. In this second sample only LM contacts with spines were considered. Of these 12 LM contacts, 7 formed synapses (there was a case where 1 bouton formed 2 synapses with 2 spines of the same dendrite), 5 did not, and 1 was unclear at EM. From these results and the initially sampled LM contacts, we calculated that the probability of an LM spine contact to form a synapse $\left(p_{\mathrm{sp}}\right)$ is 0.55 .

\section{Where are thalamic synapses located?}

In Figure $3 A$ we show the location of the dLGN LM contacts and EM-confirmed synapses in the dendrograms of all of the SSs. Most of the EM-confirmed synapses (18 of 22) were located between 50 and $100 \mu \mathrm{m}$ of path length distance to the soma (Fig. $3 B$ ). The mean path length distance of a dLGN EM-confirmed synapse to the soma is $83.6 \pm 28.3 \mu \mathrm{m}( \pm \mathrm{SD})$ and is not different from a normal distribution (Lillie test, $p=0.4818$ ). The largest distance between two dLGN EM-confirmed synapses was 110 $\mu \mathrm{m}$ (one located at $40 \mu \mathrm{m}$ and another $150 \mu \mathrm{m}$ from the soma). The average distance from soma to dendritic tip was $166 \pm 41$ 
$\mu \mathrm{m}$. The somata and proximal dendrites are spine free, and this region of the SS does not form synapses with the dLGN afferents. Since the EM-confirmed synapses were only made with spines, the earliest opportunity for the dLGN to form a synapse would be on the spine closest to the soma, which was $21 \mu \mathrm{m}$ from the soma on average. However, the closest dLGN EM-confirmed synapse was at $40 \mu \mathrm{m}$ from the soma. That the dLGN synapses do not monopolize the most proximal spines is borne out by the synaptic maps (Fig. $3 A, B$ ). These maps indicate that there is a tendency for the dLGN axons to form synapses between 50 and $100 \mu \mathrm{m}$ from the soma instead of being uniformly distributed along the dendritic tree like the LM contacts (Fig. 3C). However, this tendency does not reach statistical significance. The majority of the EM-confirmed synapses $(\sim 80 \%)$ were formed with the terminal branches of the dendrites, and the remainder with the intermediate branches.

Our sample of EM-confirmed synapses was based on prior selection through LM examination. To check whether dLGN synapses were missed by this selection, we reconstructed virtually the full length of one dendrite of neuron C0904P4C2 (indicated on the dendrograms of Fig. $3 A$ ) from serial electron microphotographs (Fig. 6). On this dendrite we did not find any synapse formed by labeled dLGN boutons that had not been documented by LM. The total length of the dendrite was $172 \mu \mathrm{m}$, and $158 \mu \mathrm{m}$ of it was reconstructed from serial EM sections. Three LM contacts were present, but only one of the three actually formed a synapse.

\section{How many thalamic synapses are formed with a spiny stellate cell?}

Previous estimates of the number of thalamic synapses onto a single neuron have relied on indirect methods (Peters and Payne, 1993; Ahmed et al., 1994; Binzegger et al., 2004). Here we have not only labeled the thalamic boutons, but by using the physical disector method (see Materials and Methods) (da Costa et al., 2009) we established for each individual SS cell the proportion of labeled dLGN boutons in the neuropil around its dendrites.

We first estimated the number of labeled dLGN synapses with each of the neurons by multiplying the number of LM contacts (Table $1, n_{\mathrm{c}}$, row 5 , Fig. 7 ) by the probability of an LM contact to form an EM-confirmed synapse (Table $1, c_{\text {syn }}$, row 6, Fig. 7). To find the total number of thalamic synapses to a single SS, we had to take into account that the neurons for which we were successful in obtaining physiology and anatomy were not in the region with the highest density of labeled dLGN terminals. Nevertheless, we could estimate their total complement of dLGN synapses by counting the number of synapses formed by labeled boutons inside a volume defined by the extent to the SS dendrites (Table 1, $d L G N_{l}$, row 3, Fig. 7) and scaling this against the proportion of all dLGN synapses in layer 4 (da Costa and Martin, 2009a).

We used the physical disector method to estimate $\mathrm{dLGN}_{1}$. Given that the labeled synapses were very sparsely distributed, we used a sampling method developed to detect rare events (da Costa et al., 2009). More than 600 disector sites were sampled in the neuropil surrounding the dendrites of each neuron to find the density of labeled synapses (row 1 in Table 1, Fig. 7). This density was then divided by the density of all dLGN synapses (row 2, Table 1, Fig. 7) to obtain $d L G N_{l}$.

With these results we could then estimate the number of dLGN synapses per neuron as well as the error of this estimation (row 7 in Table 1, Fig. 7) (see also Materials and Methods). This estimate indicated that three of the neurons formed a remarkably similar number of synapses with thalamic boutons $(\sim 130$ syn-

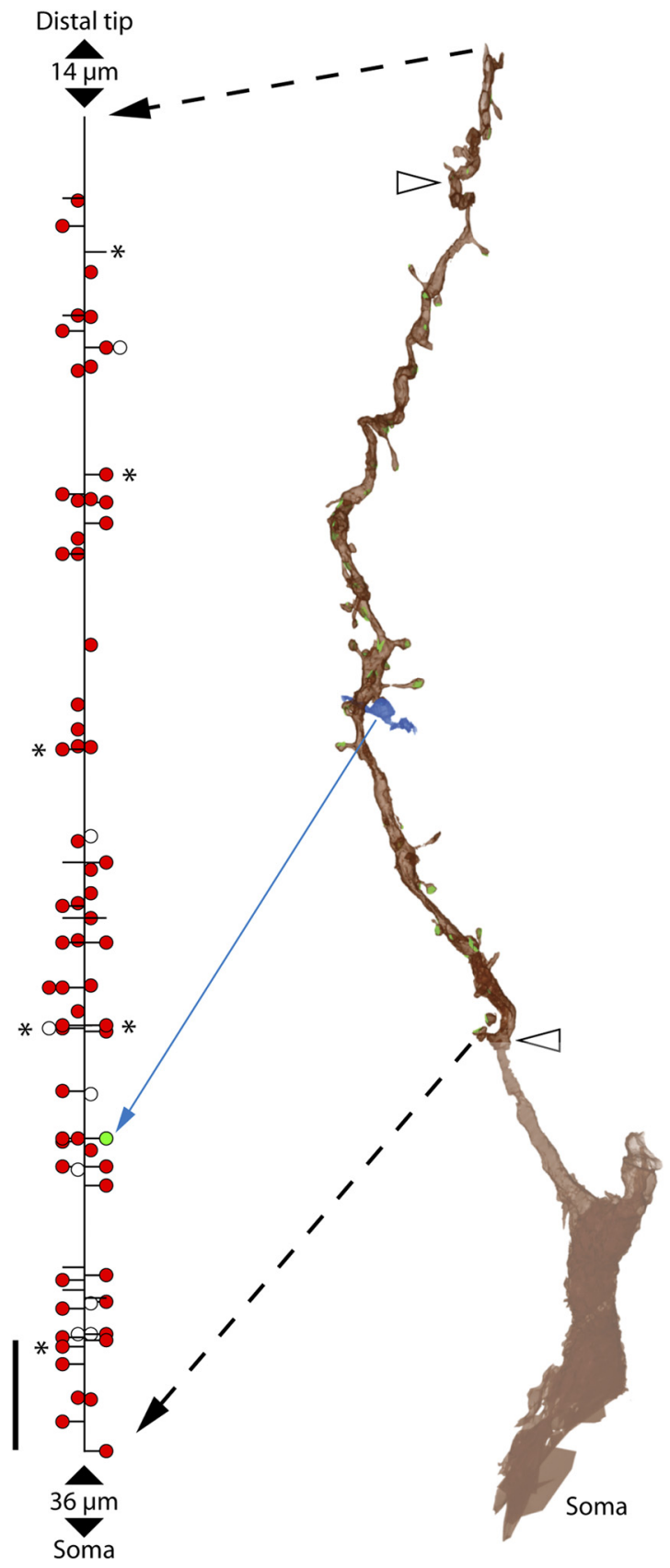

Figure 6. Serial section EM reconstruction of a spiny stellate dendrite. Right: A dLGN axon (blue) forms a synapse with the reconstructed dendrite (brown) at $65 \mu \mathrm{m}$ from the soma of neuron Cat0904-P4C2. Synapses are indicated in green. Open arrowheads indicate the locations of LM contacts that did not form a synapse. Left: Schematic summary diagram of the serially sectioned dendrite. Solid vertical line represents the dendritic shaft, solid horizontal lines indicate spines, asymmetric synapses are indicated by filled circles, symmetric synapses are indicated by open circles, and asterisks indicate spines with mitochondria. The green filled circle indicates the synapse formed with the labeled dLGN bouton; all other synapses were formed with unlabeled boutons. In spines shown without circles no synapse was found, either because they were incompletely reconstructed $(n=6)$ or because they indeed lacked synapses $(n=3)$. Vertical scale bar, $10 \mu \mathrm{m}$.

apses), while the fourth larger neuron formed a larger number (351 synapses). However, the neuron with the large number of synapses had a much larger dendritic tree than the other three (6 $\mathrm{mm}$, compared to $3.2-4.4 \mathrm{~mm}$ of the other three neurons) and was also collected from the cat with the largest mean percentage of thalamic synapses in layer 4 . 
Table 1. Estimation of the number of dLGN synapses per spiny stellate neuron

\begin{tabular}{|c|c|c|c|c|}
\hline Cell & C2003S28C1 & C0904P4C2 & C1804P3C4 & C1804P4C4 \\
\hline Row 1. Density of labeled synapses $/ \mathrm{mm}^{3}$, mean $\pm \mathrm{SEM} \times 10^{6}$ ( $n$ disectors) & $1.34 \pm 0.67(992)$ & $2.98 \pm 1.21(670)$ & $5.24 \pm 1.34(953)$ & $1.03 \pm 0.58(999)$ \\
\hline Row 2. Density of unlabeled synapses $/ \mathrm{mm}^{3}$, mean $\pm \mathrm{SEM} \times 10^{8}$ ( $n$ disectors) & $6.54 \pm 0.48(106)$ & $5.06 \pm 0.57(58)$ & $5.87 \pm 0.49(92)$ & $5.51 \pm 0.9(46)$ \\
\hline Row 3. Percentage of labeled synapses \pm error $\left(\mathrm{dLGN}_{\mathrm{l}}\right)$ & $0.21 \pm 0.1 \%$ & $0.59 \pm 0.25 \%$ & $0.89 \pm 0.24 \%$ & $0.19 \pm 0.11 \%$ \\
\hline Row 4. Percentage of dLGN synapses \pm error $\left(\mathrm{dLGN}_{\mathrm{t}}\right)^{a}$ & $2.78 \pm 0.73 \%$ & $8.15 \pm 3.15 \%$ & $5.13 \pm 1.6 \%$ & $5.13 \pm 1.6 \%$ \\
\hline Row 5. Number of contacts $\left(n_{c}\right) \pm$ error & 27 & 93 & 54 & 18 \\
\hline Row 6. Probability of a contact forming a synapse $\left(c_{\text {syn }}\right) \pm$ error & $0.33 \pm 0.19$ & $0.27 \pm 0.09$ & $0.44 \pm 0.17$ & $0.29 \pm 0.17$ \\
\hline Row 7. Estimated number of dLGN synapses \pm error & $122 \pm 99$ & $351 \pm 235$ & $138 \pm 77$ & $141 \pm 126$ \\
\hline
\end{tabular}

${ }^{a} \mathrm{da}$ Costa and Martin, 2009a.

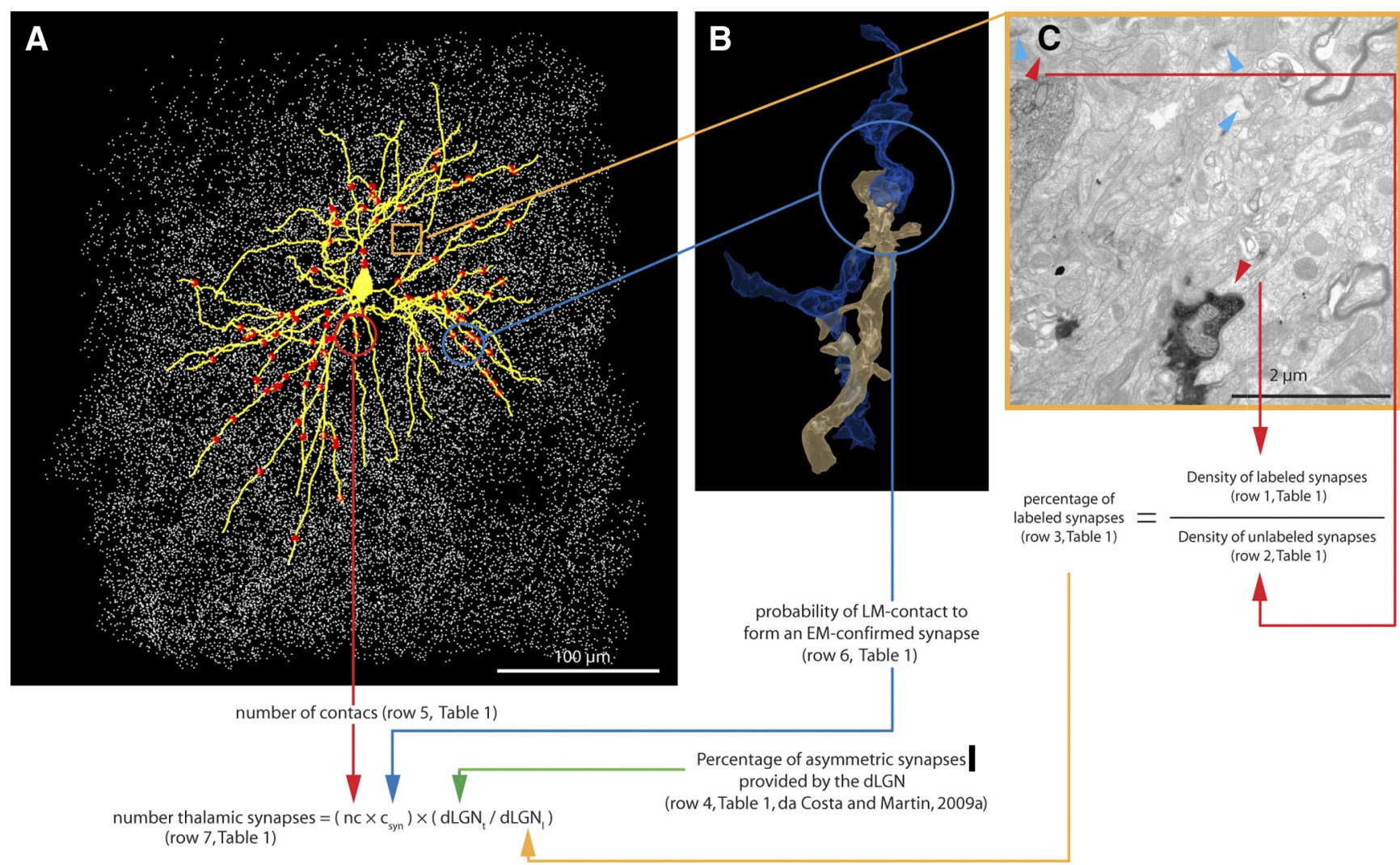

Figure 7. Experimental steps taken to calculate the total number of dLGN synapses per spiny stellate cell. This is a companion figure to Table $1 . A$, First, we calculate the total number of $L M$ contacts (red dots, $n_{c}$ ) with the dendrites (yellow) of the spiny stellate cell (see also Fig. 1A). B, Second, we verify what the probability is of an LM contact to be an EM-verified synapse (see also Figs. 4,5). $\boldsymbol{C}$, Third, we find what the density is of dLGN synapses in the vicinity of the labeled spiny stellate dendrite using the physical disector method. Shown is a disector counting frame. Synapses present in both the reference frame (data not shown) and counting frame are shown in blue, while synapses only present in the counting frame are shown in red.

In the analysis presented here, we assumed that the dLGN was the only source of labeled boutons that form the synapses counted with the disector method. The other possible source of boutons was the intracellularly labeled SS's own axon. Unfortunately, we could not rely on the reconstruction of the axonal arbors of the SS to disprove this, since the axons were incompletely filled and overlap with several branches of thalamic axons. However, previous reconstructions of SS axonal arbors have shown that in the cat the local axon of SS forms few boutons in the volume of neuropil that surrounds its own dendrite (Gilbert and Wiesel, 1979; Martin and Whitteridge, 1984; Binzegger et al., 2004) and so it is unlikely that SS synapses are a significant contamination of the several thousand boutons (Humphrey et al., $1985)$ formed by all the labeled thalamic axons.

\section{Do thalamic synapses form local clusters?}

To address this question, we measured the distance between one thalamic synapse and the nearest thalamic synapse. To increase the sample size, we also considered a proportion of the LM contacts that were formed with spines. Even though we have not investigated all of the contacts in all the neurons, we know that in this study thalamic synapses are only formed with spines of SSs and we also know the probability of a spine LM contact to be an EM-confirmed synapse $\left(p_{s p}=0.55\right)$. With this information we can then calculate the number of labeled thalamic synapses for each neuron as if we had verified at EM all of the spine LM contacts. But which of the unverified contacts are synapses? We avoided this problem by generating all possible combinations of $55 \%$ of the unverified LM spine contacts. Each of these 
combinations of spine LM contacts was then added to the pool of EMconfirmed synapses and the distance to the closest synapse was measured between them. Nineteen locations were considered for neuron Cat0904P4C2 (14 EM-confirmed synapse and 5 LM contacts), 14 for neuron Cat1804P3C4 (4 EM-confirmed synapse and 10 LM contacts), 4 for neuron Cat1804P4C4 (2 EM-confirmed synapse and 2 LM contacts), and 7 for neuron Cat2003S28C1 (2 EM-confirmed synapse and 5 LM contacts).

These real distances were then compared with distances measured from pseudorandomly located synapses on the dendrites of the same SS by using a Monte Carlo analysis. For each SS we randomly positioned thalamic synapses on its dendrites (the total number of synapses assigned to each neuron was the same as that in row 7 of Table 1). A subset of these randomly located synapses was then sampled (19 for Cat0904P4C2, 14 for Cat1804P3C4, 4 for Cat1804P4C4, and 7 for Cat2003S28C1), and the distance to the nearest next synapse was measured. Each simulation was performed 10,000 times for each SS; an example of a random distribution of thalamic synapses for each SS is shown in Figure 8.

The Monte Carlo analysis showed that for neurons Cat0904P4C2, Cat1804P4C4, and Cat2003S28C1 there was no statistical difference ( $p=0.05$ ) between the distance measurements in the real synapses and the randomly positioned ones for all possible combinations of EM-confirmed synapses and unverified LM contacts. For neuron Cat1804P3C4 there was also no statistical difference between the real synapses and the random ones for almost all combinations of unverified contacts (only 4 of 92,378 possible combinations of unverified LM contacts showed significant differences from the randomly distributed synapses).

\section{Are thalamic synapses bigger?}

When Stratford et al. (1996) studied the excitatory input to SS cells of cat area 17, they found three classes of EPSPs. The EPSPs evoked by putative dLGN axons had a larger amplitude than those of SS or layer 6 pyramidal cells. Here we assessed the size by the area of the postsynaptic density. If the size of a PSD was positively correlated with the size of an EPSP evoked by that synapse, then we would expect dLGN synapses to be larger than other synapses formed with the SS.

Fourteen of the 22 synapses formed with labeled dLGN boutons were completely reconstructed from serial EM sections, and we compared the distribution of their size with the unlabeled boutons formed with spines of the reconstructed dendrite shown in Figure $6(n=35)$. The size of synapses formed by unlabeled boutons was not significantly different from that of a normal distribution (Lillie test, $p=0.0605$ ), but the size of dLGN synapses was significantly different (Lillie test, $p=0.0087$ ). The median area of dLGN synapses formed with SS spines (median $=$

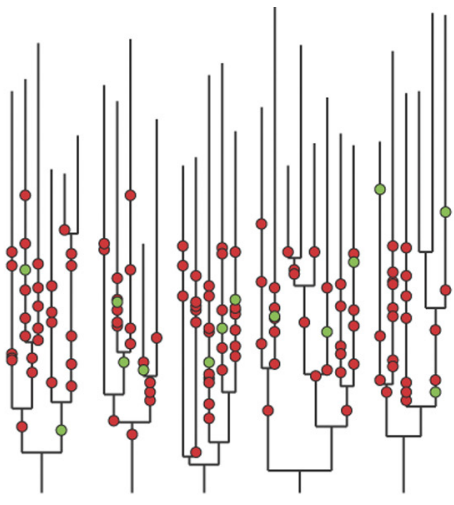

Cat1894P3C4

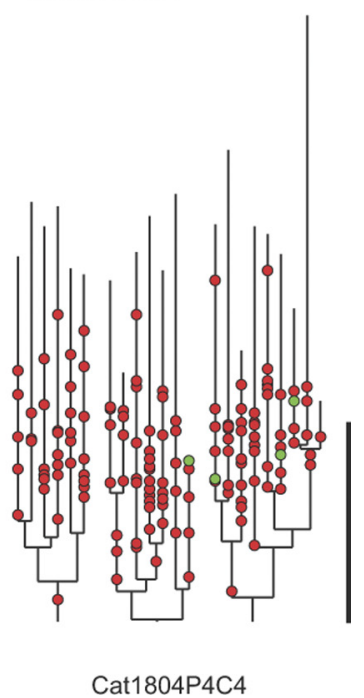

Cat0904P4C2
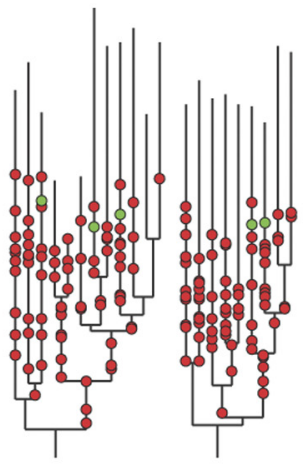

Figure 8. Randomly located synapses on the dendrites of spiny stellate cells. Dendrograms show the location of the randomly sampled, and the distance to the nearest other green location was measured and compared with the real EM-verified synapses. This process was repeated 10,000 times for each neuron.

$0.107 \mu \mathrm{m}^{2}$ ) was significantly different than the median area of spine synapses on the reconstructed dendrite (median $=0.076$ $\mu \mathrm{m}^{2}$; Wilcoxon rank sum test, $p=0.03$ ). Figure 9 shows the overlapping distributions of synapse sizes formed by both thalamic and unlabeled boutons.

\section{Discussion}

The Hubel and Wiesel (1962) model of the cat's simple cell is a cornerstone of our knowledge about cortical circuits. Yet, appealing and enduring as their model is, it is clear that we still have a very incomplete picture of the circuitry that generates the simple cell and of the thalamocortical connection in particular. In our previous biophysically detailed simulations (Banitt et al., 2007) based on our extensive studies of the structure and function of the thalamocortical link (Freund et al., 1985a,b; Anderson et al., 1994; Stratford et al., 1996) we concluded that a tight temporal control of the thalamic input was a necessary but not sufficient condition for driving simple cells. Using the same model spiny stellate cell, our simulation results have recently been confirmed by Wang et al., 2010. Here we extended our structural analyses, which led us to the inescapable fact that not only is the thalamic input numerically tiny, but there are no strong compensating structural features that might enhance the biophysical efficacy of the thalamic input to SS cells. 


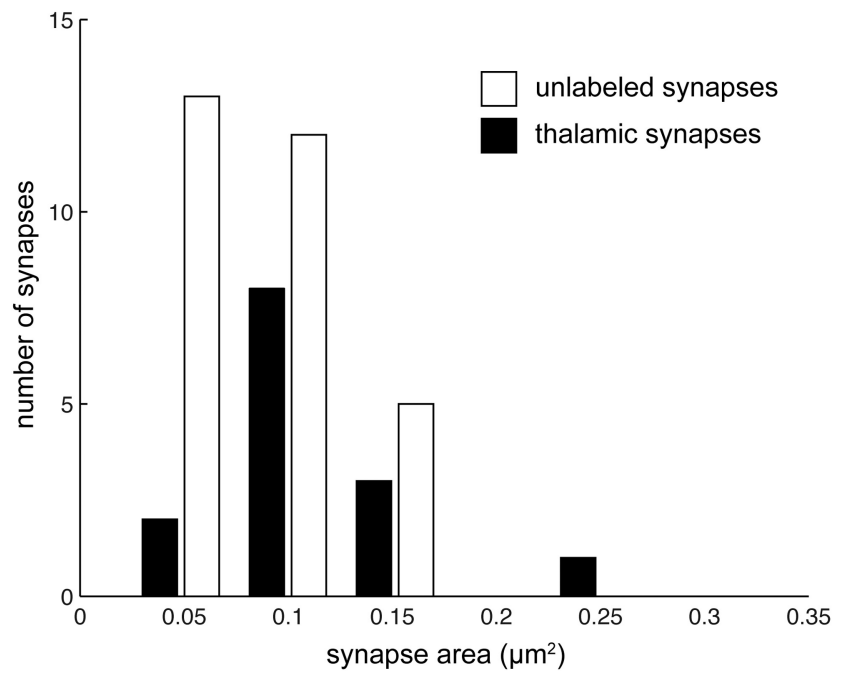

Figure 9. Histogram of the distribution of postsynaptic areas. Filled bars represent the areas of dLGN EM-confirmed synapses, and white bars represent the areas of synapses formed with spines of the dendrite shown in Figure 6.

The number of thalamic synapses formed on spiny stellate cells, its targets, and size

We found that the thalamic input that generates the multiple on and off subfields of the simple cell is delivered through between 130 and 350 synapses per target SS cell. This is a small number of synapses, $<10 \%$ of the asymmetric synapses that SSs are expected to receive (Beaulieu and Colonnier, 1985; Anderson et al., 1994; Binzegger et al., 2004), and is comparable to previous estimates for cat visual cortex: 80-100 (Binzegger et al., 2004), 100-125 (Peters and Payne, 1993), and 286 (Ahmed et al., 1994). The number of thalamocortical synapses per neuron is also small for the primate visual cortex:18-188 synapses (Peters et al., 1994; Latawiec et al., 2000). By contrast Bruno and Sakmann (2006) estimated that there were $\sim 600$ synapses per neuron in the rat barrel cortex, although they have since revised this number down to $\sim 90-580$ synapses per layer 4 neuron (Meyer et al., 2010).

We found that synapses formed by labeled dLGN boutons are significantly larger than synapses formed with the SS by unidentified boutons. This corresponds to our earlier physiological finding (Stratford et al., 1996) that the mean amplitude of a single fiber, calculated using AMPA-mediated EPSPs for the putative thalamic axons, was higher than for any other excitatory input, nearly $2.0 \mathrm{mV}$, with a strikingly small coefficient of variation (CV) of $8.3 \%$. By comparison, the SS synapses originating from other SSs had mean amplitudes of $1.0 \mathrm{mV}$ and CVs of $12-22 \%$ (Stratford et al., 1996; Tarczy-Hornoch et al., 1999). This larger amplitude EPSP of the putative dLGN afferents is not in itself enough to explain the dominance of the thalamic input, because EPSPs depressed with paired-pulse stimulation (Stratford et al., 1996). Indeed, Boudreau and Ferster (2005) have claimed that the dLGN synapses might remain relatively depressed in vivo because of their tonic activity. However, even without tonically depressed synapses, our model results indicate that the synapses will depress strongly during visual stimulation and so reduce the response of a SS (Banitt et al., 2007). The EPSP amplitudes of dLGN afferents in vivo may be more similar in average amplitude to those of the SS synapses, which show less depression (Stratford et al., 1996).

\section{The position of synapses on the dendritic tree}

Considerations of the passive cable properties of the dendrites indicate that proximal synapses are more effective than distal ones (Rall, 1967). This preferred location seems to be the case for the rodent auditory cortex assayed in vitro (Richardson et al., 2009). By contrast, our results indicate that the dLGN afferents form synapses with the SS at a mean distance from the soma of $82 \pm 26 \mu \mathrm{m}( \pm \mathrm{SD})$, which is approximately half the distance between the root and the tip of the dendrite.

Another mechanism that might boost the dLGN input is a cooperative nonlinear interaction between nearby synapses on the same dendritic tree (Shepherd et al., 1985; Polsky et al., 2004). EPSPs originating on different branches of the dendrite would summate linearly. Depending on the precise mechanism, nonlinear interactions between neighboring synapses may lead to sublinear or supralinear summation. However, our data provide no evidence for localized clustering. Instead they show that thalamic boutons form synapses with different branches and different dendrites of the same cell (Fig. 3A) and that thalamic synapses can be found at different branch levels of the dendritic tree (Fig. $3 B)$. Given the number of the thalamic synapses per neuron and the number of dendritic branches, it is statistically bound to happen that several thalamic synapses share the same dendritic segment and interact, but this appears not to be a fundamental design principle. In our study of thalamic connectivity with corticothalamic neurons (da Costa and Martin, 2009b), multiple synapses from the same axon were formed with the same dendritic segment. Here we never detected multiple synapses on a dendrite from a single axon despite the fact that SSs are surrounded by a much higher density $(\sim 10 \times)$ (Humphrey et al., 1985) of thalamic boutons than are the layer 6 corticothalamic neurons. We did, however, observe in both the SS and the corticothalamic cells one example in which a bouton formed two synapses with a dendrite.

\section{Getting to threshold and beyond}

We established that the number of dLGN synapses to single SSs is a tiny fraction of their total complement of excitatory synapses, that their location is not biophysically optimal, that the size of the synapses is large but not exceptional, that the synapses do not show any obvious tendency to cluster to promote nonlinear interactions, and that their target spines have no morphological specializations to suggest they enhance transmission to the dendrite. To make matters worse, our previous physiological studies in vitro show that they are depressing synapses (Stratford et al., 1996). All of these observations do not add up to a picture of a dominant input, yet these synapses are surely effective in linking retina to cortex. How can this be?

One solution is given by our detailed biophysical simulations (Banitt et al., 2007), which show how crucial it is that a large proportion of the EPSPs arrive at the soma within a time window to permit them to be integrated into a suprathreshold voltage (Banitt et al., 2007). Around 100 thalamic EPSPs should arrive at the soma within $10 \mathrm{~ms}$ to provide the suprathreshold depolarization (Stratford et al., 1996; Banitt et al., 2007; Douglas and Martin, 2007b; Wang et al., 2010), which is supported by functional studies of the thalamocortical pathway in cat and rat (Alonso et al., 1996; Usrey et al., 2000; Roy and Alloway, 2001; Bruno and Sakmann, 2006; Douglas and Martin, 2007b). However, it is important to note that the simulations also showed that, because of synaptic depression, this influence of the dLGN on the spike output could only be achieved with the addition of background activity arising from other cortical inputs. The presence of such 
tonic excitation is revealed by the disappearance of EPSPs when the cortex is cooled (Ferster et al., 1996).

The idea that then emerges from the anatomy, the intracellular physiology, and the modeling is that the numbers of dLGN synapses are "just enough," providing that all of the synapses deliver the required excitation "just in time" (Douglas and Martin, 2007b). It is perhaps obvious that if the number of dLGN synapses were large, then a stimulus of orthogonal orientation will also drive the simple cells, and the cardinal feature of simple cells would be lost (Banitt et al., 2007; Douglas and Martin, $2007 \mathrm{~b}$ ). Thus the mechanism of "just enough, just in time" reconciles the Hubel and Wiesel (1962) model of orientation selectivity with our evidence of the small number of dLGN synapses found on each SS.

That small numbers of neurons can significantly modulate the activity of a neuron also provides an explanation of why each neuron, with its many thousands of synapses, only fires at moderate rates even for optimal stimuli, and also why the neuronal response to natural images is so sparse. Under different stimulus conditions, different degrees of synchrony may be evoked in the different pathways that converge on a single neuron, so that although each cortical neuron receives excitatory input from many thousands of neurons (Shadlen and Newsome, 1998), it remains below threshold until sufficient synaptic charge, delivered in a tight temporal window from a few percent of the excitatory synapses, drives the neuron to spike. Whether these initial spikes die away or are amplified by local recurrent circuits is thought to depend on a "winner-take-all" mechanism (Douglas and Martin, 2007a). As with the gate of a transistor, this control of the output of a neuron by a few percent of its synapses allows for dynamic switching between the multiple afferent pathways that converge on a given neuron, thus creating a rich variety of functional subcircuits from the single hardwired cortical circuit.

\section{References}

Ahmed B, Anderson JC, Douglas RJ, Martin KA, Nelson JC (1994) Polyneuronal innervation of spiny stellate neurons in cat visual cortex. J Comp Neurol 341:39-49.

Ahmed B, Anderson JC, Martin KA, Nelson JC (1997) Map of the synapses onto layer 4 basket cells of the primary visual cortex of the cat. J Comp Neurol 380:230-242.

Alonso JM, Usrey WM, Reid RC (1996) Precisely correlated firing in cells of the lateral geniculate nucleus. Nature 383:815-819.

Anderson JC, Douglas RJ, Martin KA, Nelson JC (1994) Map of the synapses formed with the dendrites of spiny stellate neurons of cat visual cortex. J Comp Neurol 341:25-38.

Banitt Y, Martin KA, Segev I (2007) A biologically realistic model of contrast invariant orientation tuning by thalamocortical synaptic depression. J Neurosci 27:10230-10239.

Beaulieu C, Colonnier M (1985) A laminar analysis of the number of roundasymmetrical and flat-symmetrical synapses on spines, dendritic trunks, and cell bodies in area 17 of the cat. J Comp Neurol 231:180-189.

Binzegger T, Douglas RJ, Martin KA (2004) A quantitative map of the circuit of cat primary visual cortex. J Neurosci 24:8441-8453.

Botha D, Douglas RJ, Martin KA (1987) TRAKA: a microcomputer-assisted system for digitizing the three-dimensional structure of neurones. J Physiol 394:16P.

Boudreau CE, Ferster D (2005) Short-term depression in thalamocortical synapses of cat primary visual cortex. J Neurosci 25:7179-7190.

Bruno RM, Sakmann B (2006) Cortex is driven by weak but synchronously active thalamocortical synapses. Science 312:1622-1627.

Cardona A, Saalfeld S, Preibisch S, Schmid B, Cheng A, Pulokas J, Tomancak P, Hartenstein V (2010) An integrated micro- and macroarchitectural analysis of the Drosophila brain by computer-assisted serial section electron microscopy. PLoS Biol 8:e1000502.

Chung S, Ferster D (1998) Strength and orientation tuning of the thalamic input to simple cells revealed by electrically evoked cortical suppression. Neuron 20:1177-1189.

Colonnier M (1968) Synaptic patterns on different cell types in the different laminae of the cat visual cortex: an electron microscope study. Brain Res 9:268-287.

da Costa NM, Martin KA (2009a) The proportion of synapses formed by the axons of the lateral geniculate nucleus in layer 4 of area 17 of the cat. J Comp Neurol 516:264-276.

da Costa NM, Martin KA (2009b) Selective targeting of the dendrites of corticothalamic cells by thalamic afferents in area 17 of the cat. J Neurosci 29:13919-13928.

da Costa NM, Hepp K, Martin KA (2009) A systematic random sampling scheme optimized to detect the proportion of rare synapses in the neuropil. J Neurosci Methods 180:77-81.

Douglas RJ, Martin KA (2007a) Recurrent neuronal circuits in the neocortex. Curr Biol 17:R496-R500.

Douglas RJ, Martin KA (2007b) Mapping the matrix: the ways of neocortex. Neuron 56:226-238.

Ferster D, Chung S, Wheat H (1996) Orientation selectivity of thalamic input to simple cells of cat visual cortex. Nature 380:249-252.

Fiala JC (2005) Reconstruct: a free editor for serial section microscopy. J Microsc 218:52-61.

Freund TF, Martin KA, Whitteridge D (1985a) Innervation of cat visual areas 17 and 18 by physiologically identified X- and Y- type thalamic afferents. I. Arborization patterns and quantitative distribution of postsynaptic elements. J Comp Neurol 242:263-274.

Freund TF, Martin KA, Somogyi P, Whitteridge D (1985b) Innervation of cat visual areas 17 and 18 by physiologically identified X- and Y-type thalamic afferents. II. Identification of postsynaptic targets by GABA immunocytochemistry and Golgi impregnation. J Comp Neurol 242:275-291.

Garey LJ, Powell TP (1971) An experimental study of the termination of the lateral geniculo-cortical pathway in the cat and monkey. Proc R Soc Lond B Biol Sci 179:41-63.

Gilbert CD, Wiesel TN (1979) Morphology and intracortical projections of functionally characterised neurones in the cat visual cortex. Nature 280:120-125.

Gray EG (1959) Axo-somatic and axo-dendritic synapses of the cerebral cortex: an electron microscope study. J Anat 93:420-433.

Gundersen HJ, Jensen EB (1987) The efficiency of systematic sampling in stereology and its prediction. J Microsc 147:229-263.

Hamos JE, Van Horn SC, Raczkowski D, Sherman SM (1987) Synaptic circuits involving an individual retinogeniculate axon in the cat. J Comp Neurol 259:165-192.

Hornung JP, Garey LJ (1981) The thalamic projection to cat visual cortex: ultrastructure of neurons identified by Golgi impregnation or retrograde horseradish peroxidase transport. Neuroscience 6:1053-1068.

Hubel DH, Wiesel TN (1962) Receptive fields, binocular interaction and functional architecture in the cat's visual cortex. J Physiol 160:106-154.

Humphrey AL, Sur M, Uhlrich DJ, Sherman SM (1985) Projection patterns of individual X- and Y-cell axons from the lateral geniculate nucleus to cortical area 17 in the cat. J Comp Neurol 233:159-189.

Latawiec D, Martin KA, Meskenaite V (2000) Termination of the geniculocortical projection in the striate cortex of macaque monkey: a quantitative immunoelectron microscopic study. J Comp Neurol 419:306-319.

LeVayS (1986) Synaptic organization of claustral and geniculate afferents to the visual cortex of the cat. J Neurosci 6:3564-3575.

LeVay S, Stryker MP, Shatz CJ (1978) Ocular dominance columns and their development in layer IV of the cat's visual cortex: a quantitative study. J Comp Neurol 179:223-244.

Martin KA, Whitteridge D (1984) Form, function and intracortical projections of spiny neurones in the striate visual cortex of the cat. J Physiol 353:463-504

McGuire BA, Hornung JP, Gilbert CD, Wiesel TN (1984) Patterns of synaptic input to layer 4 of cat striate cortex. J Neurosci 4:3021-3033.

Meyer HS, Wimmer VC, Hemberger M, Bruno RM, de Kock CP, Frick A, Sakmann B, Helmstaedter M (2010) Cell type-specific thalamic innervation in a column of rat vibrissal cortex. Cereb Cortex 20:2287-2303.

Peters A, Payne BR (1993) Numerical relationships between geniculocortical afferents and pyramidal cell modules in cat primary visual cortex. Cereb Cortex 3:69-78. 
Peters A, Payne BR, Budd J (1994) A numerical analysis of the geniculocortical input to striate cortex in the monkey. Cereb Cortex 4:215-229.

Polsky A, Mel BW, Schiller J (2004) Computational subunits in thin dendrites of pyramidal cells. Nat Neurosci 7:621-627.

Rall W (1967) Distinguishing theoretical synaptic potentials computed for different soma-dendritic distributions of synaptic input. J Neurophysiol 30:1138-1168.

Richardson RJ, Blundon JA, Bayazitov IT, Zakharenko SS (2009) Connectivity patterns revealed by mapping of active inputs on dendrites of thalamorecipient neurons in the auditory cortex. J Neurosci 29: 6406-6417.

Roy SA, Alloway KD (2001) Coincidence detection or temporal integration? What the neurons in somatosensory cortex are doing. J Neurosci 21: 2462-2473.

Sanderson KJ (1971) The projection of the visual field to the lateral geniculate and medial interlaminar nuclei in the cat. J Comp Neurol 143: 101-108.

Shadlen MN, Newsome WT (1998) The variable discharge of cortical neurons: implications for connectivity, computation, and information coding. J Neurosci 18:3870-3896.

Shepherd GM, Brayton RK, Miller JP, Segev I, Rinzel J, Rall W (1985) Signal enhancement in distal cortical dendrites by means of interactions between active dendritic spines. Proc Natl Acad Sci U S A 82:2192-2195.
Slomianka L, West MJ (2005) Estimators of the precision of stereological estimates: an example based on the CAl pyramidal cell layer of rats. Neuroscience 136:757-767.

Sterio DC (1984) The unbiased estimation of number and sizes of arbitrary particles using the disector. J Microsc 134:127-136.

Stratford KJ, Tarczy-Hornoch K, Martin KA, Bannister NJ, Jack JJ (1996) Excitatory synaptic inputs to spiny stellate cells in cat visual cortex. Nature 382:258-261.

Tarczy-Hornoch K, Martin KA, Stratford KJ, Jack JJ (1999) Intracortical excitation of spiny neurons in layer 4 of cat striate cortex in vitro. Cereb Cortex 9:833-843.

Usrey WM, Alonso JM, Reid RC (2000) Synaptic interactions between thalamic inputs to simple cells in cat visual cortex. J Neurosci 20:5461-5467.

Wang HP, Spencer D, Fellous JM, Sejnowski TJ (2010) Synchrony of thalamocortical inputs maximizes cortical reliability. Science 328 : $106-109$

White EL, Rock MP (1980) Three-dimensional aspects and synaptic relationships of a Golgi-impregnated spiny stellate cell reconstructed from serial thin sections. J Neurocytol 9:615-636.

Winfield DA, Powell TP (1983) Laminar cell counts and geniculo-cortical boutons in area 17 of cat and monkey. Brain Res 277:223-229. 